\title{
Proton induced thermal stress-wave measurements using a Laser Doppler Vibrometer
}

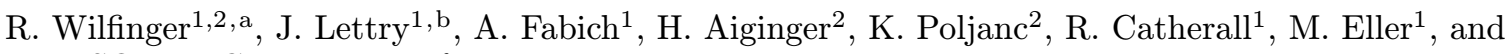 \\ The ISOLDE Collaboration \& Technical team \\ 1 CERN, European Organization for Nuclear Research, 1211 Genève 23, Switzerland \\ 2 Atominstitut, Vienna University of Technology, Stadionallee 2, 1020 Vienna, Austria
}

Received: January 31, 2007

\begin{abstract}
Thermal stress-waves are generated in the solid target material when the proton beam interacts. These stress waves excite natural oscillations of the target or cause plastic deformations. Hence, an experimental setup with a laser Doppler vibrometer [1] was developed to investigate free surface vibrations of cylindrical targets. The target configurations for RIB and conventional neutrino beams (CNGS project) were investigated to analyze proton induced thermal stress-wave generation and propagation.
\end{abstract}

PACS. 42.62.Cf Industrial laser applications - 42.68.Wt Remote sensing - 42.79.Qx Laser Doppler velocimeters -43.40 . $+\mathrm{s}$ Structural acoustics and vibration - 46.40.-f Vibrations and mechanical waves $-62.30 .+\mathrm{d}$ Mechanical and elastic waves; vibrations

\section{Introduction}

Radioactive ion beams (RIB) and neutrino beams are generated by the nuclear interaction of a proton beam in a target. The production of high secondary particle intensities require high-power primary proton beams, which cause stress formations up to the fatigue limit or even up to the structural strength of solid targets.

Thermal stress-waves are generated in the solid target material when the proton beam interacts. These stress waves excite natural oscillations of the target or cause plastic deformations. Hence, an experimental setup with a laser Doppler vibrometer (LDV) was developed to investigate free surface vibrations of cylindrical targets. The target configurations for RIB and conventional neutrino beams (CNGS project $[2,3]$ ) were investigated to analyze proton induced thermal stress-wave generation and propagation.

The chosen measurement device allowed to measure individually the immediate response after proton impact. Additionally, the transient state and the following natural vibrations of the eigenmodes with its decay behavior were analyzed. Surface displacement amplitudes in the sub-micrometer range could be recorded at distances of up to $40 \mathrm{~m}$ without any contact between the target and the sensor.

In the following, the LDV and the experimental setup are described. The goal of this experiment was to apply the laser Doppler vibrometry technique to measure

\footnotetext{
a e-mail: Roman.Wilfinger@cern.ch

b e-mail: Jacques.Lettry@cern.ch
}

proton induced thermal stress waves in solid targets of different target geometries and materials. The CNGS carbon-composite target rod was chosen to investigate the basic LDV principle of stress wave measurements for highpower targets. On the other hand, high- $Z$ materials are used as a spallation neutron source in ISOLDE target units. Autopsy of these target units showed major deformations and displacements of strongly fixed Ta and W proton-neutron converters, which caused electrical shortcuts or unacceptable reductions of the desired radioisotope yields. Therefore, carbon-composite and tantalum were chosen as materials as representative target materials for this experiment. The measured characteristic free surface velocity and displacement signals for cylindrical targets irradiated with a proton beam parallel to their axis are given and discussed.

\section{Laser Doppler Vibrometer}

In principle, laser Doppler vibrometers [1] measure the instantaneous velocity and displacement on vibrating surfaces from the Doppler shift in the frequency of backscattered laser light. Unlike conventional non-contact methods which measure only the absolute value of the velocity, LDV's are able to detect changes in direction and even stationary conditions by using the heterodyne detection method [4]. Therefore, the LDV permits measurement of even hot surfaces without any mass-loading, leaving the vibration properties of the object under investigation unchanged. 


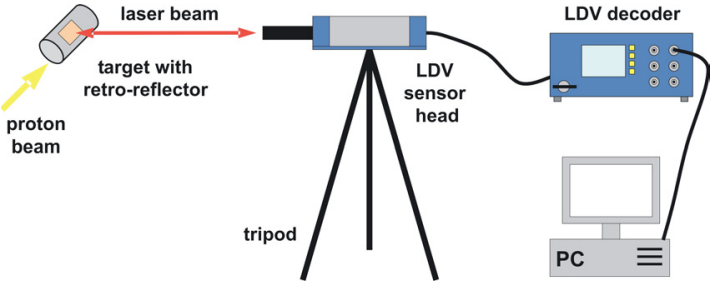

Fig. 1. Schematic illustration of the LDV setup.

In a LDV sensor head, the beam of a helium neon laser with a wavelength of $\lambda=632.8 \mathrm{~nm}$ is split into a reference beam and a measurement beam. The measurement beam is focused onto the object surface under investigation. The reflected beam is then merged with the reference beam onto the detector, where a fringe pattern typical for interferometry is generated. One complete fringe cycle on the detector corresponds to an object displacement of exactly half of the wavelength of the used laser light.

Additionally, when laser light is reflected on a moving surface, the frequency of the reflected light is Dopplershifted and the frequency change is directly proportional to the velocity of the moving surface. The direction of the velocity can be determined by introducing an additional fixed frequency shift in the interferometer to which the Doppler shift is added with the correct sign. This heterodyne principle allows to identify the direction of the object movement and to measure also at zero velocity for out-of-plane vibrations, where only motions parallel to the laser beam are detected.

An industrial single point laser Doppler vibrometer from PolyteC C), Germany, has been used for the measurements (figure 1). The optical sensor head OFV-505 is connected with a data cable to the OFV-5000 modular vibrometer controller which contained the decoder units. The VD-02 wide-bandwidth velocity decoder with a resolution of $0.15 \mu \mathrm{m} / \mathrm{s}, 10 \mathrm{~m} / \mathrm{s}$ maximum velocity and $1.5 \mathrm{MHz}$ upper frequency limit was connected to a PC data acquisition card containing a $40 \mathrm{MHz}$ two-channel analyzer. The sensor head itself consists of a laser source, an interferometer, an exchangeable optical lens for different focal lengths and a photo-detector. To get the highest possible signal-to-noise ratio, the laser beam has to be optimally focused. The vibrometer can be optimally adapted to different ranges of stand-off distances by using different front lenses for the sensor head.

The laser vibrometer is capable to study the characteristics of mechanical vibrations or transient motion processes. The velocity information is extracted from the frequency modulation of the Doppler signal, while the displacement signal can be reconstructed from the phase modulation available at the same time. In the case of harmonic vibrations the velocity and displacement signal provide equivalent information. In contrast to this, transient motion sequences in most cases are shown more clearly by the displacement signal. If the intensity of the reflected light is very low, it is better to choose the velocity signal because of the independence of the frequency shift from intensity variations. Occasional signal dropouts can be avoided by using a tracking filter during the measurement or by filtering out manually after the measurement.

The absolute noise limited resolution of the velocity decoder is approximately $0.2 \mu \mathrm{m} / \mathrm{s} / \sqrt{\mathrm{Hz}}$ [5]. This gives a value of $0.3 \mathrm{pm}$ for the displacement for a corresponding amplitude of a sinusoidal vibration of $100 \mathrm{kHz}$. This means that with high frequencies, a significantly higher resolution can be attained with velocity acquisition than with displacement decoders. The maximum vibration frequency of the displacement decoder is limited to about $250 \mathrm{kHz}$. Hence, for applications at higher frequencies, velocity recording is the only option.

It is possible to increase the reflectivity of a surface using retro-reflective tape or paint. This material consists of small glass spheres (approximately $50 \mu \mathrm{m}$ in diameter) that are coated at the rear face with a reflective layer and glued with an elastic epoxy to the base material. Each sphere acts as a small "cat's eye" scattering light back along the path of the incident beam. The incident light is returned to the LDV aperture over an angle of a couple of degrees. As the laser beam usually hits several glass beads at one time each of the reflected beams can interfere with each other and produce a granular pattern of intensity as the distances travelled differ by a small amount. This intensity pattern is called a "speckle pattern". As most targets are not perfectly stationary, the LDV will not stay within one bright speckle during the measurement. The detector will see different dark and bright speckles. As the speckles have different phases this mechanism causes noise in the laser vibrometer output. The speckle noise produced depends on the rate of change in the speckle pattern and is therefore strong when measuring with out-of-plane LDV motions on surfaces that show a strong in-plane motion (and vice versa). The signal shows in this case single spikes, which are only effects produced by speckle "dropouts".

\section{Experimental setup}

The experiment was situated in the ISOLDE [6] target area. The CERN Proton Synchrotron Booster (PSB) [7] beam is sent to the ISOLDE front-ends with a nominal kinetic energy of $1.4 \mathrm{GeV}$ and a maximum of about $32 \times 10^{12}$ protons distributed over four bunches with a total pulse length of about $2.4 \mu \mathrm{s}$. The proton beam at the focal plane was Gaussian shaped in both transverse planes with a radius of $2.2 \mathrm{~mm}$ (r.m.s.). The proton beam position on target could be varied either in horizontal and/or in vertical direction.

The sensor head was placed behind concrete shielding and the laser beam was deflected via a remote controlled front surface mirror $10 \mathrm{~m}$ from the target, as shown in figure 2. Thin retro-reflection tapes were glued onto the target surface to increase the intensity of the reflected laser light. The LDV sensor head was mounted on small table with a micro-step motor (figure 3 ) to tilt the plate with the sensor head in a vertical direction to adjust the height of the laser beam. 


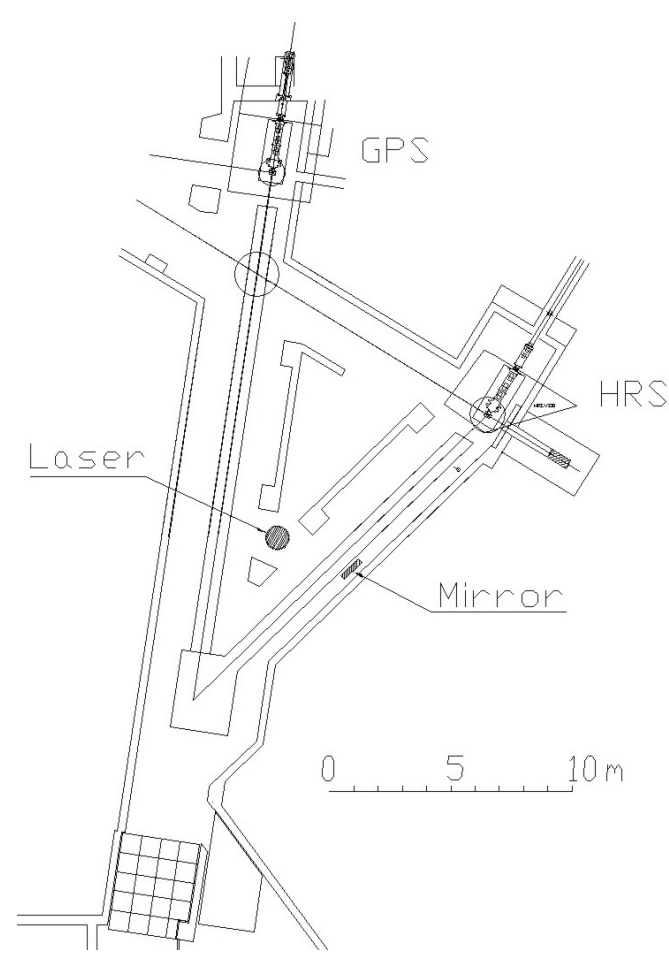

Fig. 2. Map of the ISOLDE prime area indicating the position of the LDV sensor head between the robot rails, the first surface coated mirror and the HRS front-end.

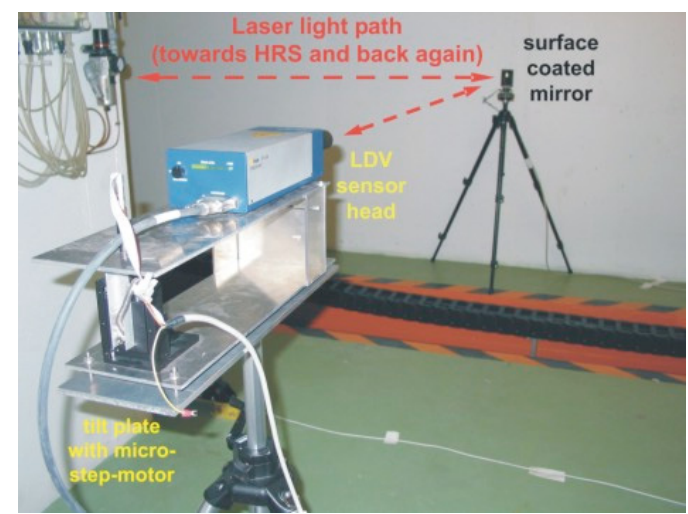

Fig. 3. LDV sensor head on a tilt plate directed to a surface coated mirror to deflect the laser beam towards the HRS front-end where the targets were placed during the measurement. The mirror was placed on a remotely controlled rotation platform to scan the laser beam along the cylinder.

With this setup the free-surface velocity was measured for different solid target cylinders after a single proton pulse impact delivered by the PSB.

\section{Targets and target-mount}

The target and target support were constructed to facilitate a fast exchange and a precise alignment of different target materials with cylindrical geometries. Precise positioning of the proton beam according to the target axis and the generation of well-defined vibration modes of the

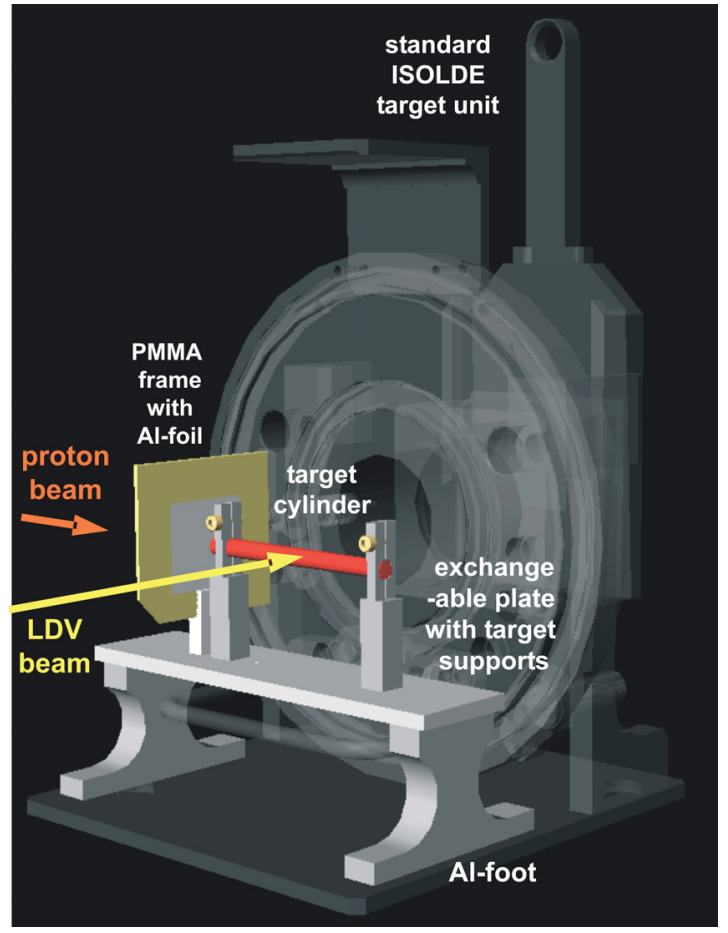

Fig. 4. Drawing of the support for cylindrical target used at ISOLDE HRS front-end.

target after proton beam impact had to be ensured. Ambiguous vibrations caused by undefined suspension of the target had to be reduced to a minimum.

The targets used during the experiments at ISOLDE were mounted on a standard ISOLDE target- and ionsource unit without neither a target-container nor an ion source, which is shown schematically in figure 4 . The center of the target cylinders and discs were placed in the PSB proton beam focal plane at the middle of the target base. The cylinders and discs were jammed in the hole of the support poles. The position of the proton beam was evaluated by the activation of a $5 \mu \mathrm{m}$ thick Al-foil which was placed about $80 \mathrm{~mm}$ upstream of the HRS focal plane, as indicated in figure 4 . The position of the proton beam was verified by measuring the position of maximum activation.

The targets used during the experiments at ISOLDE had cylindrical geometry and were made of Ta and C (carbon composite used for CNGS target). Depending on the length of cylinders we distinguished between discs $(\mathrm{L} \leq \mathrm{d})$ and cylinders $(\mathrm{L} \gg \mathrm{d})$, where the diameter $d$ was always $10 \mathrm{~mm}$, except for the CNGS target rod [2] with $d=5 \mathrm{~mm}$. The cylindric target shapes reduced multiple stress wave reflections on the surfaces and allowed independent analysis and identification of different vibration modes. The radial symmetry also allows application of one-dimensional models for the radial stress waves.

\section{Experimental results}

All speckle dropouts were removed manually from the recorded free surface velocity signals. The displacement 


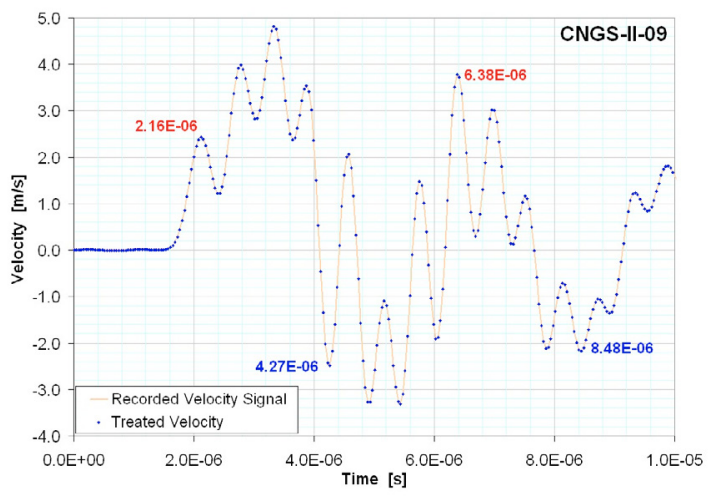

Fig. 5. Recorded free-surface velocity signal generated by radial stress waves inside a CNGS target rod. Details of the first $10 \mu \mathrm{s}$. The proton pulse for this signal had a total length of $2.3 \mu \mathrm{s}$ and consisted of four equally distributed bunches with a total intensity of $26.8 \times 10^{12}$ protons. The origin of the time scale was chosen arbitrarily.

signal was calculated by numerical integration from this treated velocity signal. The total target response is basically a composition of the excitation by the proton pulse, the transient state and the superposition of many different natural oscillation modes with corresponding damping constants. The latter are a valuable input for mechanical design of high-power proton targets.

\subsection{Elastic stress wave propagation inside the CNGS target-rod}

The generation and propagation of elastic radial stress waves inside a CNGS target rod was investigated during the experiments. The proton pulse had a total length of $2.4 \mu \mathrm{s}$ and consisted of four almost equally distributed bunches with a total intensity of $26.8 \times 10^{12}$ protons. The proton beam cross-section covered the whole target cross section and caused a radial compression wave that could be detected at the surface. The first radial target response has the same time structure as the impacting proton pulse as shown in the free-surface velocity history in figure 5 . The compression stress wave is transformed into a tension wave during reflection at a free surface. This tension wave is moving to the opposite side of the cylinder and its counterpart arrives after $2.1 \mu \mathrm{s}$ at the surface with the probing laser spot. The arrival of this tension wave can be seen by a decrease in the velocity signal. The $5 \mathrm{~mm}$ target diameter and the repeated $2.1 \mu$ s time intervals correspond to the $2381 \mathrm{~m} / \mathrm{s}$ sound velocity in the used carbon composite material. The corresponding propagation of the stress waves in a one-dimensional model is illustrated in a time/position diagram, given in figure 6 .

The axis of the CNGS target rod was able to move within a circle of $100 \mu \mathrm{m}$ diameter around the center of the target support hole. Thus, the CNGS target rod can be considered having free ends. With free ends, different vibrations and oscillation modes such as bending, rolling

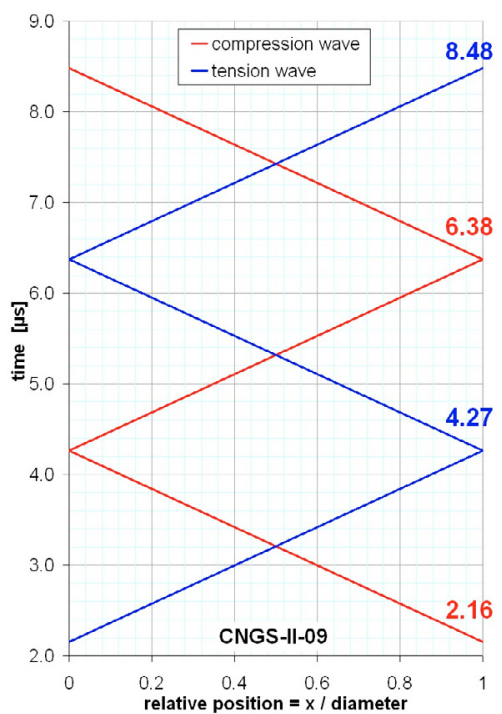

Fig. 6. $(x, t)$-diagram for the propagation of cylindrical radial stress waves inside a CNGS target rod, schematically illustrated in a one-dimensional model by two elastic waves, which are named after their stress character at the first response at the LDV spot position. The time marker correspond to the free-surface velocity history given in figure 5 .

or bouncing of the whole target rod inside the support holes could be observed. For the rolling motion, a characteristic frequency of $f \approx 70 \mathrm{~Hz}$ was obtained from a circular pendulum model. This is in excellent agreement with the observed time period found in all CNGS surface displacement histories of $14.2 \mathrm{~ms}$, which corresponds to a frequency of $70 \mathrm{~Hz}$.

About $50 \mu$ s after the proton impact, the target starts to oscillate in its natural bending mode. This particular bending oscillation is always superimposed to any rolling or bouncing of the rod inside the support holes. The bending mode of the CNGS target rod given in figure 7 has a time period of $0.946 \mathrm{~ms}$ which corresponds to a frequency of $1057 \mathrm{~Hz}$ with a 1/e-damping constant of about $3.3 \mathrm{~ms}$. Thus, the bending oscillation is damped below $5 \%$ of its initial value after $10 \mathrm{~ms}$. This value is confirmed by figure 7 where the bending oscillation is already deadened after $5 \mathrm{~ms}$.

\subsection{Elastic stress wave propagation in tantalum cylinders}

For the tantalum cylinder, a single bunch with an intensity of about $7.0 \times 10^{12}$ protons and a pulse length of $0.23 \mu \mathrm{s}$ was chosen. Hence, the time for a stress wave to cross the tantalum cylinder diameter is longer than the applied proton-pulse length. The beam radius was $2.2 \mathrm{~mm}$ (r.m.s.), hence smaller than the $5 \mathrm{~mm}$ target radius. Therefore, a steep thermal gradient was generated inside the target material immediately after proton pulse impact.

In this situation, four different stress waves are generated (in a one-dimensional model), as shown in figure 8 . 


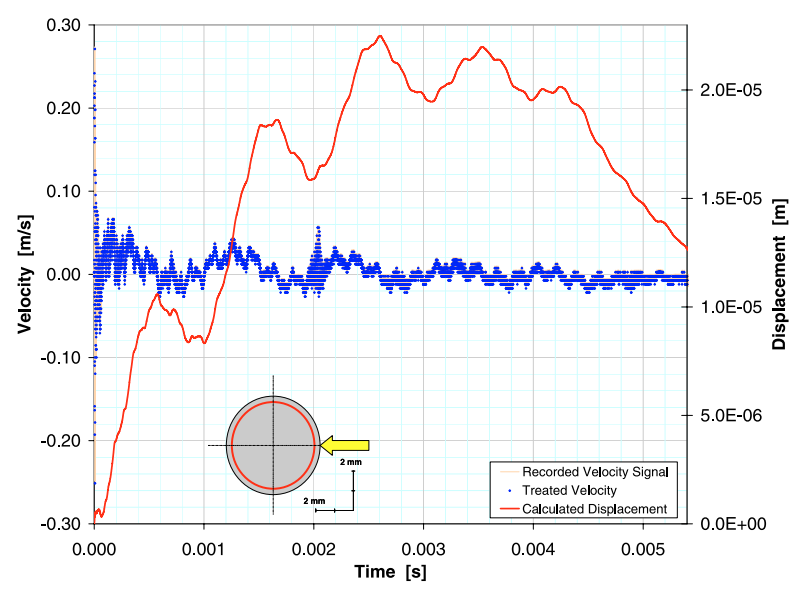

Fig. 7. Recorded free-surface velocity signal generated by radial stress waves. Details of the first $5 \mathrm{~ms}$. The proton pulse for this signal had a total length of $2.3 \mu \mathrm{s}$ and consisted of four equally distributed bunches with a total intensity of $26.8 \times 10^{12}$ protons. Schematic sketch in the diagram: The red circle indicates the position of the proton beam cross-section in relation to the target cross-section. The arrow points at the radial measurement position.

The stress waves are numbered and named according to their first arrival and character (compression or tension) at the surface with the probing laser spot. The first stress wave (1) is a compression wave, which is generated at the outside of the thermic hot region closer to the surface with the probing laser spot. The second stress wave (2) is a tension wave, generated inside the thermic hot region at the opposite side of the proton beam channel. The third stress wave (3) is created as a compression wave, which is transformed into a tension wave after reflection at the free surface opposite to the probing laser spot. The fourth stress wave (4) is generated as a tension wave at the same position as the first wave that propagates in the opposite direction. It is then transformed into a compression wave after reflection at the rear target surface.

The free-surface velocity history of the four different radial stress waves arrivals at the target surface with the laser spot is shown in figure 9. The first response of the target clearly indicates the arrival of a compression wave (1), a tension wave (2) and the converted compression wave as a tension wave (3) and the transformed tension wave as a compression wave (4). Due to multiple reflections of these radial stress waves inside the tantalum cylinder the situation becomes complex and the reflected waves cannot be clearly identified after further reflections. Additionally, an increasing time gap between the two compression peaks (1 and 4) was observed when the proton beam axis was displaced horizontally.

The response of a Ta-disc was qualitatively identical to the Ta cylinder, except for the missing bending oscillation at approximately $1.89 \mathrm{kHz}$, as shown in figure 10 . The proton pulse impact generated stress waves and excited different natural oscillation modes with various damping constants could be obtained from the full width at half maximum of the corresponding frequency peak in the

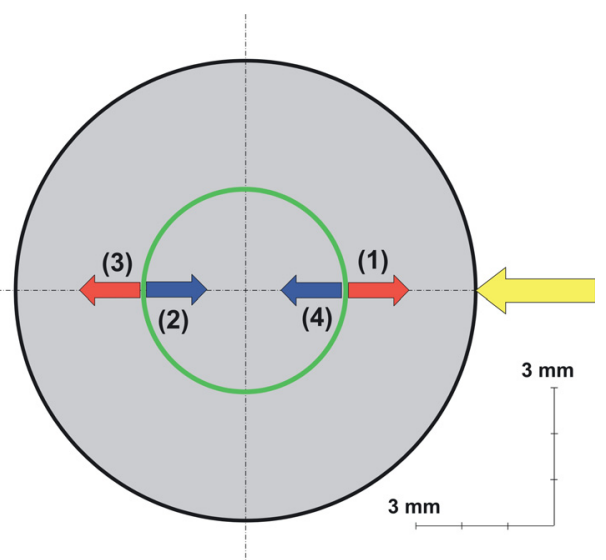

Fig. 8. Schematic illustration of the radial stress waves generated in a cylindrical target immediately after proton beam impact for a proton-pulse length which is shorter than the time for a stress wave to cross the target diameter. The red circle indicates the position of the proton beam cross-section in relation to the target cross-section. The arrow points at the radial measurement position.

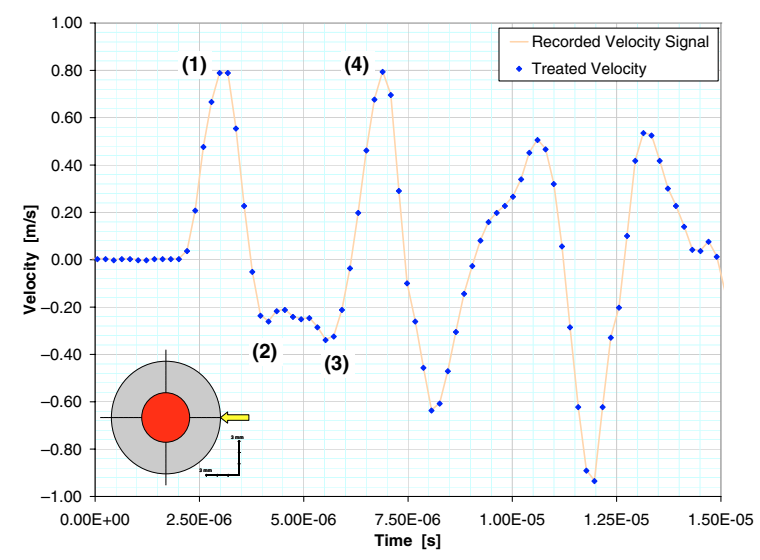

Fig. 9. Free-surface velocity signal for an axially centered proton beam showing the generation of cylindrical radial stress waves inside a tantalum cylinder. The proton pulse for this signal had a total length of $0.23 \mu \mathrm{s}$ and consisted of one single bunch with a total intensity of $7.6 \times 10^{12}$ protons. The origin of the time scale was chosen arbitrarily. Schematic sketch in the diagram: The red circle indicates the position of the proton beam cross-section in relation to the target cross-section. The arrow points at the radial measurement position.

FFT-spectra. The observed transient time was longer for an asymmetric excitation by an axially displaced proton beam compared to axially centered pulses. Only elastic stress waves were observed for the tantalum cylinder and disc after an impact of a proton pulse with a maximum intensity of $28.0 \times 10^{12}$ protons per pulse.

\section{Summary}

The results of these thermal stress wave experiments showed the successful use of a laser Doppler vibrometer 


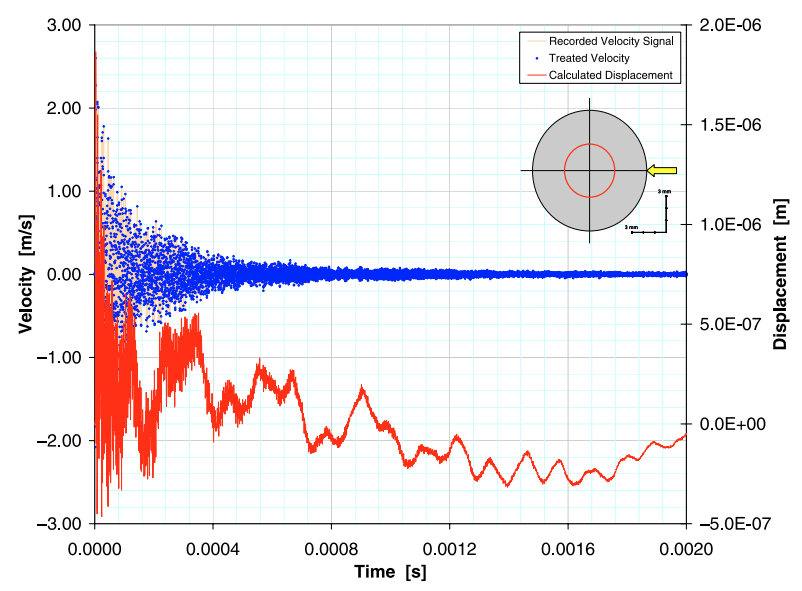

Fig. 10. Free-surface velocity signal measured on a $15 \mathrm{~mm}$ long tantalum cylinder for a $2 \mathrm{~mm}$ vertically displaced proton beam. The corresponding displacement signal obtained by integration of the velocity signal. Details of the first $2 \mathrm{~ms}$. The proton pulse for this signal had a total length of $2.3 \mu$ s and consisted of four equally distributed bunches with a total intensity of $28.0 \times 10^{12}$ protons. The origin of the time scale was chosen arbitrarily.

device to measure proton induced thermal stress wave generation and propagation in high-power proton targets in harsh environments. The only condition is that the laser beam has unhindered optical access to the object. For this reason careful attention has to be paid to the reflectivity of the object-surface, its direction of vibration and the reduction of the signal losses on the laser light path. The described measurement device and setup will be used to further improve the proton induced effects on solid targets.

This work was performed within the "Austrian Doctoral Student Programme" at CERN, instituted and financed by the Austrian Federal Ministry for Education, Science and Culture.

\section{References}

1. Polytec Webpage, http://www.polytec.com (2005)

2. M. Buhler-Broglin, K. Elsener (eds.), L.A. Lopez Hernandez, G.R. Stevenson, M. Wilhelmsson, General Description of the CERN Project for a Neutrino Beam to Gran Sasso (CNGS), CERN AC Note (2000-2003), ISBN 92-9083-174-X (2000)

3. CNGS Webpage, http://proj-cngs.web.cern.ch/proj-cngs, $11 / 02 / 2002$

4. M.K. Mazumder, Appl. Phys. Lett. 16, 462 (1970)

5. Private communication with technical team of PolyteC ${ }^{(C), ~ h t t p: / / w w w . p o l y t e c . c o m ~(2004) ~}$

6. E. Kugler, The ISOLDE facility, Hyperfine Interact. 129, $23(2000)$

7. K.H. Reich, Proc. 3rd National Particle Accelerator Conference PAC 3, Washington, DC, US, 1969 (1969), pp. 959-961 